\title{
Photon generation in ferromagnetic point contacts
}

\author{
A.M. Kadigrobov ${ }^{1,2}$, R.I. Shekhter ${ }^{1}$, and M. Jonson ${ }^{1,3,4}$ \\ ${ }^{1}$ Department of Physics, University of Gothenburg, Gothenburg SE-412 96, Sweden \\ E-mail: kadig@tp3.ruhr-uni-bochum.de \\ ${ }^{2}$ Theoretische Physik III, Ruhr-Universität Bochum, Bochum D-44801, Germany \\ ${ }^{3}$ SUPA, Institute of Photonics and Quantum Sciences, Heriot-Watt University, Edinburgh EH14 4AS, Scotland, UK \\ ${ }^{4}$ Department of Physics, Division of Quantum Phases and Devices, Konkuk University, Seoul 143-701, Korea
}

Received June 20, 2012

\begin{abstract}
We show theoretically that a significant spin accumulation can occur in electric point contacts between two ferromagnetic electrodes with different magnetizations. Under appropriate conditions an inverse population of spin-split electronic levels results in stimulated emission of photons in the presence of a resonant electromagnetic field. The intensity of the emitted radiation can be several orders of magnitude higher than in typical semiconductor laser materials for two reasons. (1) The density of conduction electrons in a metal point conduct is much larger than in semiconductors. (2) The strength of the coupling between the electron spins and the electromagnetic field that is responsible for the radiative spin-flip transitions is set by the magnetic exchange energy and can therefore be very large as suggested by Kadigrobov et al. [Europhys. Lett. 67, 948 (2004)].
\end{abstract}

PACS: 73.40.-c Electronic transport in interface structures.

Keywords: spin-flip transitions, ferromagnetic point contacts, electromagnetic field, photon emission.

\section{Introduction}

Typical metals are characterized by a high density of conduction electrons compared with other types of conductors. At the same time metals are linear electrical conductors in the sense that their response to an external electrical field is linear in the field strength. This means that it is very difficult to realize a highly nonequilibrium distribution of electrons in an ordinary metal. It is known, e.g., that strong electric fields inside a metal usually lead to melting due to Joule heating rather than to a significant excitation of the conduction electrons. Metal point contacts are remarkable exceptions because of a spatial separation between the small volume in which the external power is transferred to the electrons and the much larger region where this power is dissipated. This is the case for nanometer sized microconstrictions (microbridges) joining two bulk metals, where the size of the constriction volume where the electrical current density can be very high - is much smaller than the inelastic relaxation length of the electrons [1-3]. Unlike in bulk metals, current densities in such constrictions can reach $10^{7}-10^{10} \mathrm{~A} / \mathrm{cm}^{2}$ without any significant heating of the material [4].

Point-contact spectroscopy is a well known method for studying phonons and other excitations in metals through their interaction with the "hot" electrons created in the constriction. Electrical point contacts made of magnetic metals bring a new dimension to point-contact spectroscopy through the possibility to study the influence of the electron's spin degree of freedom on charge transport. Spin dependent tunneling and the possibility to inject "hot" spin-polarized electron's into a point-contact have opened up the possibility to locally (on the nanometer length scale) control the magnetic ordering of the material $[5,6]$. In addition, by electrically controlled thermal heating of metals, fresh opportunities for thermoelectrically manipulating nanomagnets have been demonstrated [7-9].

A significant nonequilibrium spin accumulation together with a highly inverted population of the spin-split electron levels such as may occur in nanosized heterocontacts between nonidentical magnets or in point contacts between a magnetic and a normal metal subject to an external magnetic field [10-14] offers a new area of research. Such an inverse level population makes it possible for the electrons to relax by a radiative spin-flip transition from the upper to the lower spin level as a photon is emitted.

This paper is devoted to a detailed theory of stimulated light emission due to radiative electron spin-flip transitions in ferromagnetic point contacts. We will show that the optical gain $g_{\text {opt }}$ in a single point contact can reach values of $10^{7}-10^{8} \mathrm{~cm}^{-1}$, which is orders of magnitude higher than in the best semiconductor laser materials. As discussed in 
Ref. 14 a laser device based on arrays of such point contacts is expected to have a gain of $10^{6}-10^{7} \mathrm{~cm}^{-1}$. The high gain is due to an unconventional mechanism for the interaction between a spin and an electromagnetic field based on the magnetic exchange interaction and briefly discussed in Ref. 10. The exchange interaction also leads to a large spin-level splitting and hence to a high frequency of the emitted radiation.

\section{2. "Exchange-orbital" interaction between free electron spin and electromagnetic field}

The Hamiltonian for electrons in a magnetically ordered conductor can be written as

$$
\hat{H}=\hat{H}_{0}(\hat{\mathbf{p}}, \mathbf{r}) \hat{\sigma}_{0}-\sigma \cdot \mathbf{I} .
$$

Here $\hat{H}_{0}(\hat{\mathbf{p}}, \mathbf{r})$ is the Hamiltonian of an electron in the absence of the exchange interaction, $\hat{\mathbf{p}}$ is the electron momentum operator, $\mathbf{r}$ is the electron coordinate, $\hat{\sigma}_{0}$ is the $2 \times 2$ unity matrix, $\sigma=\left(\sigma_{x}, \sigma_{y}, \sigma_{z}\right)$ are Pauli matrices, and $\mathbf{I}$ is a vector directed along the magnetization with a magnitude given by the exchange energy. According to Eq. (1) the dispersion law for electrons with spins up/down is

$$
E_{\uparrow \downarrow}(\mathbf{p})=\varepsilon(\mathbf{p}) \mp \mathbf{I},
$$

where $\varepsilon(\mathbf{p})$ is eigenvalue of the spin-independent Hamiltonian $\hat{H}_{0}(\hat{\mathrm{p}}, \mathbf{r})$.

The spin-split energy levels presented in the above equation allows a resonant electron-photon interaction in which a photon is emitted or absorbed by an electron undergoing a spin-flip. As the photon momentum is negligibly small, the electron momentum does not change in such a process and hence a radiative electron transition between the spin up $E_{\uparrow}$ and spin down $E_{\downarrow}$ energy bands is vertical. According to Eq. (2) the energy conservation law for vertical transition of electrons with emission of photons of frequency $\omega$ does not depend on the electron momentum:

$$
E_{\downarrow}(\mathbf{p})-E_{\uparrow}(\mathbf{p})-\hbar \omega=2 I-\hbar \omega=0 .
$$

It follows that if $\omega=2 I / \hbar$ all "hot" electrons are in resonance with the electromagnetic field, and hence in a ferromagnetic metal with an inverse population of electrons (which can be created by injection of a spin-polarized current $[10,11,15,16])$, stimulated emission of light due to transitions of electrons from filled states in the upper band to empty states in the lower band is possible.

Conventional mechanisms of interaction of electron spins with an electromagnetic field are the Zeeman interaction and spin-orbit interaction

$$
\begin{gathered}
E_{Z}=g \mu_{B} \mathbf{h}_{e m} \cdot \sigma, \quad \mu_{B}=\frac{e \hbar}{2 m c}, \\
E_{s-\text { orb }}=\frac{e \hbar}{m^{2} c^{2}}\left(\mathbf{p} \times \mathcal{E}_{e m}\right) \cdot \sigma,
\end{gathered}
$$

where $g$ is the gyromagnetic ratio, $\mu_{B}$ is the Bohr magneton, $e, m$ are the electron charge and mass, respectively, $c$ is the velocity of light, and $\mathbf{h}_{e m}$ and $\mathcal{E}_{e m}$ are the magnetic and electric components of the electromagnetic field. Both of these interactions are weak due to their relativistic nature. However, for practical applications it is desirable to use low magnetic and electric fields, and hence to have stronger interactions of the electromagnetic field with the spin.

For ferromagnetic conductors we suggest a more effective "spin-orbital-exchange" mechanism for the direct interaction between the electromagnetic field and the spins of conduction electrons [10]. This mechanism is electrostatic in origin and based on the dependence of the exchange interaction (between conduction and localized electrons) on the conduction electron momentum $\mathbf{p}$ (see, e.g., Ref. 17).

This exchange interaction, being a many-body effect, has to do with the overlap of the wave functions of the conduction electrons and the magnetic subsystem. A change of the conduction electron momentum affects the overlap and results in a change of the exchange energy. Therefore, the exchange energy in the Hamiltonian (1) may be written as $\mathbf{I}(\hat{\mathbf{p}})$, where in the presence of an electromagnetic field described by a vector potential A the momentum operator $\hat{\mathbf{p}}$ must be changed to $\hat{\mathbf{p}}-(e / c) \mathrm{A}$.

Expanding the exchange interaction to linear order in A, the Hamiltonian (1) is transformed into an effective Hamiltonian

$$
\hat{H}_{\mathrm{eff}}=\hat{H}_{p}+\hat{H}_{\mathrm{eff}}^{(1)}
$$

where the perturbation term can be written as

$$
\hat{H}_{\mathrm{eff}}^{(1)}=\frac{e}{2 c} \sigma \cdot\left(A_{i} \frac{\partial \mathbf{I}(\hat{\mathbf{p}})}{\partial \hat{p}_{i}}+\frac{\partial \mathbf{I}(\hat{\mathbf{p}})}{\partial \hat{p}_{i}} A_{i}\right),
$$

if we omit the term

$$
\hat{\sigma}_{0} \frac{e}{m c} \mathbf{A} \cdot \hat{\mathbf{p}}
$$

which does not flip spins (summation over repeated indices is implied).

Using the effective Hamiltonian (6) one finds the energy of an electron in a magnetically ordered conductor under an electromagnetic field to be

$$
E_{e m}^{\sigma}=E_{\sigma}(\mathbf{p})+E_{\text {ex-orb }},
$$

where

$$
E_{\sigma}(\mathbf{p})=\sigma_{0} \varepsilon(\mathbf{p})-\sigma \cdot \mathbf{J}(\mathbf{p})
$$

The second term in Eq. (8),

$$
E_{\text {ex-orb }}=\sigma \cdot \frac{\partial \mathbf{I}}{\partial p_{i}} \frac{e}{c} A_{i},
$$


couples the spin and the momentum of the electron, and being proportional to the exchange interaction gives rise to a relatively large peculiar spin-orbital interaction - "exchange spin-orbital interaction". Taking $A=(c / \omega) h_{e m}$ and estimating the derivative in Eq. (10) as $\partial I / \partial p_{i} \sim I / p_{0}$ where $p_{0}=\hbar / a \approx m v_{F}$ ( $m$ is the mass of a free electron, $v_{F}=10^{8} \mathrm{~cm} / \mathrm{s}$ is the Fermi velocity, and $a$ is an atomic length) one obtains

$$
E_{\text {ex-orb }} \approx \frac{c}{v_{F}} \mu_{B} h_{e m}
$$

which means that the exchange-spin-orbital interaction is two orders of magnitude stronger than the Zeeman interaction Eq. (4).

If the injected electrons are prepared in such a way that their spins are not parallel to the magnetization in the active region, the Hamiltonian (6) produces spin-flips and hence stimulates radiative transitions of hot electrons from the upper to the lower energy band.

A microcontact between two different magnetic metals results in a "focusing" of injected hot electrons in spin space - an effect known as spin-dependent electron tunneling [18-21]. Due to this effect an excess spin density is accumulated in the point-contact region in conjunction with a concentration of the current. Under certain conditions such an accumulation is accompanied by an inverse populations of the spin-split energy levels $[10,11,15,16]$. If this is the case, we show that electron-photon scattering results in generation of coherent photons, which leads to an enhancement of the electromagnetic field propagating through the point contact. This effect has been observed experimentally [11]. Recently, photon emission from ferromagnetic metal junctions was also observed [22,23].

In Sec. 3 below we will outline the formalism to be used for calculating the electrical current through a point contact.

\section{Formulation of the problem}

The system under consideration is a point contact between two ferromagnetic metals (labelled 1 and 2 in Fig. 1) under electromagnetic irradiation. The exchange energies and magnetization directions in the metals are assumed to be different as is illustrated in Fig. 1. We furthermore consider the point contact to be in the diffusive transport regime, so that the elastic mean free path $l_{0}$ is much shorter than the characteristic size $d$ of the contact.

In order to calculate the electrical current through the point contact (PC) one needs to find the distribution functions $f_{\sigma \mathbf{p}}^{(1,2)}(\mathbf{r})$ of electrons in metals 1 and 2 to the left and right of the constriction, respectively (see Fig. 1), where $\mathbf{p}$ is the electron momentum and $\sigma= \pm 1$ is the electron spin. In each of the metals these functions satisfy the Boltzmann equations:

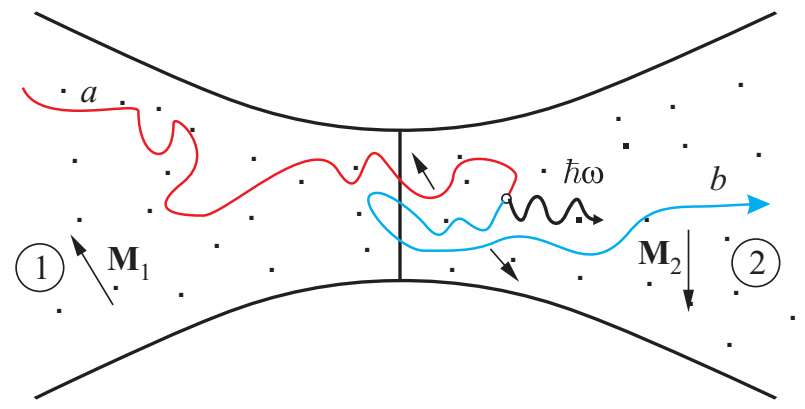

Fig. 1. (Color online) Diffusive point contact under irradiation. A voltage bias $V$ injects a spin-polarized current from ferromagnetic metal 1 with magnetic moment $\mathbf{M}_{1}$ into ferromagnetic metal 2 with magnetic moment $\mathbf{M}_{2}$. A spin-up electron is shown to move along a diffusive trajectory from metal 1 to metal 2 (red line $a$ ) where it resonantly interacts with the electromagnetic field, which results in a spin-flip and the emission of a photon. Continuing along its diffusive path with spin down (blue line $b$ ) the spin-dependent contact resistance implies that the radiation induced spin-flip contributes to a change of the magnetoresistance of the point contact.

$$
\begin{gathered}
\mathbf{v}_{\sigma}^{(s)} \frac{\partial f_{\sigma \mathbf{p}}^{(s)}}{\partial \mathbf{r}}-e \frac{\partial \Phi^{(s)}}{\partial \mathbf{r}} \frac{\partial f_{\sigma \mathbf{p}}^{(s)}}{\partial \mathbf{p}}+\frac{f_{\sigma \mathbf{p}}^{(s)}-\left\langle f_{\sigma \mathbf{p}}^{(s)}\right\rangle_{s}}{t_{\sigma}^{(s)}}= \\
=\sigma w_{\mathbf{p h}}^{(s)}\left\{f_{\uparrow \mathbf{p}}^{(s)}, f_{\downarrow \mathbf{p}}^{(s)}\right\}, \quad s=1,2 .
\end{gathered}
$$

Here $\mathbf{v}_{\sigma}^{(s)}(\mathbf{p})$ is the electron velocity in each metal, which is given by a momentum derivative of the electron energy as $\mathbf{v}_{\sigma}^{(s)}=\partial E_{\sigma}^{(s)}(\mathbf{p}) / \partial \mathbf{p}$, where

$$
E_{\sigma}^{(1,2)}(\mathbf{p})=\varepsilon_{1,2}(\mathbf{p})-\sigma \mathbf{I}_{1,2}(\mathbf{p})
$$

are the electron dispersion laws inside metals 1 and $2 ; \mathbf{I}_{1,2}$ are the electron exchange energy in metal 1 and 2 , $t_{\sigma}^{(s)}=l_{0} /\left|\mathbf{v}_{\sigma}^{(s)}\right|$ is the electron free path time and $l_{0}$ is the electron elastic relaxation length, $\Phi^{(s)}$ is the electric potential; the notation $\langle\ldots\rangle_{S}$ implies an average of the bracketed quantity over the relevant Fermi surface,

$$
\langle\ldots\rangle_{s}=\int \frac{d S_{\mathbf{p}}}{\left|\mathbf{v}^{(s)}\right|}(\ldots) / \int \frac{d S_{\mathbf{p}}}{\left|\mathbf{v}^{(s)}\right|} .
$$

The electrical potential $\Phi^{(s)}(\mathbf{r})$ can be found from the electrical neutrality condition,

$$
\sum_{\sigma}\left\langle f_{\sigma \mathbf{p}}^{(s)}(\mathbf{r})-n_{F}\left(E_{\sigma}^{(s)}(\mathbf{p})\right)\right\rangle_{s}=0,
$$

supplemented with the boundary conditions

$$
\Phi^{(s)}\left(z \rightarrow(-1)^{s} \infty\right)=(-1)^{s+1} V / 2,
$$

where $n_{F}$ is the Fermi function and $V$ is the bias voltage.

In the case that the electromagnetic field has a large amplitude the interaction of electrons with photons may be 
treated semi-classically, which allows the electron-photon collision integral to be written as

$$
\begin{gathered}
w_{\mathrm{ph}}^{(s)}\left\{f_{\uparrow \mathbf{p}}^{(s)}, f_{\downarrow \mathbf{p}}^{(s)}\right\}=\frac{2 \pi}{\hbar}\left|W_{s}\right|^{2}\left[f_{\mathbf{p}-\mathbf{q} \downarrow}^{(s)}-f_{\uparrow \mathbf{p}}^{(s)}\right] \times \\
\times \frac{\pi^{-1} v_{s f}}{\left(E_{\uparrow}^{(s)}(\mathbf{p})-E_{\downarrow}^{(s)}(\mathbf{p}-\mathbf{q})-\hbar \omega\right)^{2}+v_{s f}^{2}} .
\end{gathered}
$$

Here $W_{s}=\left\langle\Psi_{i}\left|\hat{H}_{\mathrm{eff}, s}^{(1)}\right| \Psi_{f}\right\rangle$ is the matrix element corresponding to the transition of an electron in metal $s$ from the initial state $\Psi_{i}$ (corresponding to the initial energy $E_{i}$ ) to the final state $\Psi_{f}$ (corresponding to the final energy $E_{f}$ ), which describes the coupling between the electron spin and an electromagnetic field of frequency $\omega$ and momentum q; Hamiltonian $\hat{H}_{\mathrm{eff}, s}^{(1)}$ is given by Eq. (7) with the change $\mathbf{I}(\hat{\mathbf{p}}) \rightarrow \mathbf{I}_{s}(\hat{\mathbf{p}})$. While writing Eq. (17) we assumed the energy $\delta$-function to be broadened due to the spin-flip processes of the spin relaxation rate $v_{s f}$. According to Eqs. (7) and (11), the modulus of the matrix element $W_{s}$ is equal to

$$
\left|W_{s}\right|=b\left|\left[\mathbf{e}_{1} \cdot \mathbf{e}_{2}\right]\right| \frac{c}{v_{F}} \frac{I_{s}}{\hbar \omega} \mu_{B} h_{e m},
$$

where the constant $b \sim 1$, the unit vectors $\mathbf{e}_{1}$ and $\mathbf{e}_{2}$ are directed along the magnetization in metals 1 and 2 .

To facilitate explicit calculations we will consider a simplified geometry, approximating the point contact by a cylindrical channel of length $L$ and diameter $d$, with $L \gg d \gg l_{0}$. The boundary conditions for electron distribution functions $f_{\sigma \mathbf{p}}^{(s)}$ at the interface between metals 1 and 2 can be written as

$$
\begin{gathered}
f_{\sigma \mathbf{p}}^{(1)}=\left(1-D_{\sigma}\right) f_{\sigma \mathbf{p}_{R}}^{(1)}+D_{\sigma} f_{\sigma \overline{\mathbf{p}}}^{(2)}, \\
f_{\sigma \mathbf{p}}^{(2)}=D_{\sigma} f_{\sigma \mathbf{p}_{R}}^{(1)}+\left(1-D_{\sigma}\right) f_{\sigma \overline{\mathbf{p}}}^{(2)} .
\end{gathered}
$$

Here $D_{\sigma}=D_{\sigma}(\mathbf{p}, \overline{\mathbf{p}})$ is the spin-dependent transparency of the interface; $\mathbf{p}=\left(\mathbf{p}_{\|}, p_{z}\right)$ and $\mathbf{p}_{R}=\left(\mathbf{p}_{\|},-p_{z}\right)$ (here $p_{\|}=\left(\left(p_{x}, p_{y}\right)\right)$, the $z$ axis is along the channel $)$ are the momenta of the incident and reflected electrons, respectively; the momentum of the transmitted electron $\overline{\mathbf{p}}$ is determined by the energy conservation condition, $E_{\sigma}^{1,2}(\mathbf{p})=E_{\sigma}^{2,1}(\overline{\mathbf{p}})$.

Away from the contact region, the current spreads over a large volume so that its density decreases and the electron system is essentially in equilibrium at distances $|\mathbf{r}| \gg d$. As $d \ll L$ we will use the additional boundary conditions $f_{\sigma \mathbf{p}}^{(1,2)}(z= \pm L / 2)=n_{F}\left(E_{\sigma}^{(1,2)}(\mathbf{p})\right)$.

We consider the limit of a weak electron-photon scattering $d / l_{\text {ph }} \ll 1$, where $l_{\text {ph }}$ is the electron-photon scattering length. In this limit it is possible to solve the Boltzmann equation. This allows us to solve the Boltzmann equation (12) by perturbation theory expanding $w_{\mathrm{ph}}^{(s)}, f_{\sigma \mathbf{p}}^{(s)}$, and $\Phi^{(s)}$ in powers of the small parameter $d / l_{\mathrm{ph}}$.
In order to solve the kinetic equation (12) to zeroth order in $d / l_{\mathrm{ph}}$ we generalize the procedure developed in Refs. 3, 24, 25 to allow for spin dependent electron dynamics. To zeroth order, the distribution functions $f_{\sigma \mathbf{p}}^{(s)}$ can be written as

$$
\begin{aligned}
& f_{\sigma \mathbf{p}}^{(s)}=\alpha_{\sigma \mathbf{p}}^{(s)} n_{F}\left(E_{\sigma}^{(s)}(\mathbf{p})+e \phi_{0}(\mathbf{r})-e V / 2\right)+ \\
& +\left(1-\alpha_{\sigma \mathbf{p}}^{(s)}\right) n_{F}\left(E_{\sigma}^{(s)}(\mathbf{p})+e \phi_{0}(\mathbf{r})+e V / 2\right),
\end{aligned}
$$

where $\alpha_{\sigma \mathbf{p}}^{(s)}(\mathbf{r})$ is the probability that an electron emanating from far inside the ferromagnet $(z=-\infty)$ diffuses elastically to reach point $\mathbf{r}$ in metal $s$ with momentum $\mathbf{p}$, the concrete form of the electrical potential $\phi_{0}(\mathbf{r})$ inside the point contact is not important in the limit $e V \ll \varepsilon_{F}$. The distribution functions $f_{\sigma \mathbf{p}}^{(2)}$ are sketched in Fig. 2.

To linear approximation in the parameter $l_{0} / d \ll 1$, it follows from Eqs. (12) and (19) that this probability can be written as $\alpha_{\sigma \mathbf{p}}^{(s)}=\left\langle\alpha_{\sigma \mathbf{p}}^{(s)}\right\rangle-l_{0}\left(v_{z} /|\mathbf{v}|\right) d \alpha_{\sigma \mathbf{p}}^{(s)} / d z$. The isotropic part of $\alpha_{\sigma \mathbf{p}}^{(s)}$ satisfies the diffusion equation

$$
\frac{d^{2}}{d z^{2}}\left\langle\alpha_{\sigma \mathbf{p}}^{(s)}\right\rangle=0,
$$

with the boundary conditions $\left\langle\alpha_{\sigma \mathbf{p}}^{(1)}(z=-L / 2)\right\rangle=1$ and $\left\langle\alpha_{\sigma \mathbf{p}}^{(2)}(z=L / 2)\right\rangle=0$; in the vicinity of the interface between the two magnetic metals the effective boundary conditions are $[3,11]$

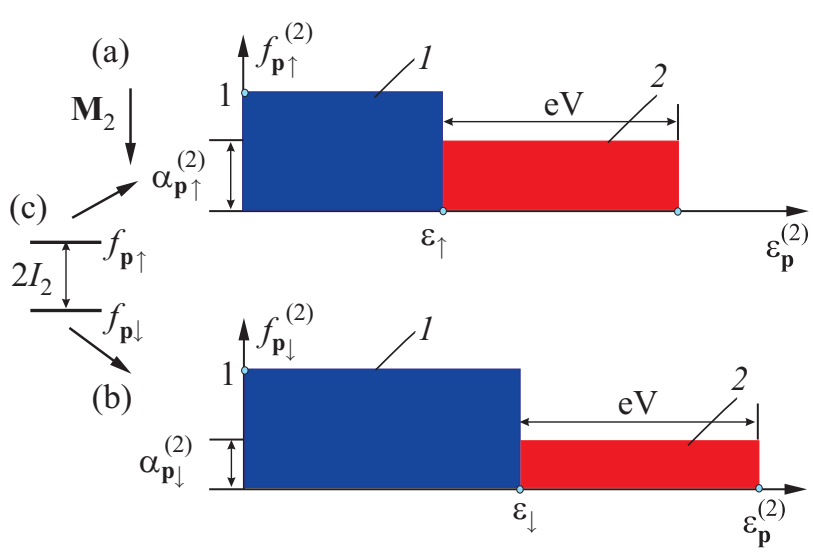

Fig. 2. (Color line) Zero-temperature energy distributions for (a) magnetic moment-up (spin-down), $f_{\mathbf{p} \uparrow}$, and (b) magnetic moment-down (spin-up) electrons, $f_{\mathbf{p} \downarrow}$, at point $\mathbf{r}$ in ferromagnetic metal 2 of the point contact. The inset (c) shows the Zeeman energy splitting and the magnetization direction $\mathbf{M}_{2}$. All states are occupied up to $\varepsilon_{\uparrow}=\varepsilon_{f}-e V / 2-I$ and $\varepsilon_{\downarrow}=\varepsilon_{f}-e V / 2+I$, respectively (blue rectangles, 1 ), but in the intervals $\left(\varepsilon_{\uparrow}, \varepsilon_{\uparrow}+e V\right)$ and $\left(\varepsilon_{\downarrow}, \varepsilon_{\downarrow}+e V\right)$ the states are only partly occupied (red rectangles, 2 ) and to an extent that is determined by the probabilities $\alpha_{\uparrow \mathbf{p}}(\mathbf{r})$ and $\alpha_{\downarrow \mathbf{p}}(\mathbf{r})$ for "hot" electrons in the ferromagnetic metal to reach $\mathbf{r}$. Clearly, the difference between the densities of spin-down and spin-up electrons, $n_{\uparrow}(\mathbf{r})-n_{\downarrow}(\mathbf{r}) \propto\left[\left(\alpha_{\uparrow}^{(2)}-\alpha_{\downarrow}^{(2)}\right) e V-2 I\right]$, depends on the bias voltage $V$. It follows that the spin population can be inverted, so that $n_{\uparrow}(\mathbf{r})>n_{\downarrow}(\mathbf{r})$, for large enough $V$ if $\alpha_{\uparrow}^{(2)}>\alpha_{\downarrow}^{(2)}$. 


$$
\begin{gathered}
\left\langle\alpha_{\sigma \mathbf{p}}^{(2)}\right\rangle-\left\langle\alpha_{\sigma \mathbf{p}}^{(1)}\right\rangle=\frac{l_{0}}{\left\langle D_{\sigma}\right\rangle} \frac{d\left\langle\alpha_{\sigma \mathbf{p}}^{(1)}\right\rangle}{d z} ; \\
\frac{d\left\langle\alpha_{\sigma \mathbf{p}}^{(1)}\right\rangle}{d z}=\frac{d\left\langle\alpha_{\sigma \mathbf{p}}^{(2)}\right\rangle}{d z}
\end{gathered}
$$

if the transparency of the interface is assumed to be small, $\left\langle D_{\sigma}\right\rangle \ll 1$. Solving the diffusion equation (21) with these boundary conditions one finds

$$
\left\langle\alpha_{\sigma}^{(1)}\right\rangle=1-\beta_{\sigma}^{(1)}\left(1+\frac{2 z}{L}\right) ; \quad\left\langle\alpha_{\sigma}^{(2)}\right\rangle=\beta_{\sigma}^{(2)}\left(1-\frac{2 z}{L}\right),
$$

where

$$
\beta_{\sigma}^{(s)}=\frac{\kappa_{\sigma}^{(s)}}{1+\kappa_{\sigma}^{(1)}+\kappa_{\sigma}^{(2)}} ; \quad \kappa_{\sigma}^{(s)}=\left\langle D_{\sigma}\right\rangle \frac{L}{2 l_{0}} .
$$

\section{Spin accumulation in the point contact}

If electrons are injected from metal 1 (i.e., if $e V>0$ ) the numbers of "hot" electrons with spin up and down are

$$
\begin{gathered}
\delta n_{\sigma}=\int_{\Omega_{P C}^{(2)}} d^{3} \mathbf{r} \int \frac{d^{3} \mathbf{p}}{(2 \pi \hbar)^{3}} \times \\
\times\left[f_{\mathbf{p} \sigma, 0}^{(2)}(\mathbf{r})-n_{F}\left(E_{\sigma}^{(2)}(\mathbf{p})+e \Phi_{0}(\mathbf{r})+\frac{e V}{2}\right)\right],
\end{gathered}
$$

where $\Omega_{P C}^{(2)}$ is the volume of the normal-metal part of the contact ("active" zone). Using Eqs. (20), (21), and (23) in Eq. (25) one finds

$$
\delta n_{\sigma}=\frac{\beta_{\sigma}^{(2)}}{4}\left(n_{0} \Omega_{P C}^{(2)}\right) \frac{e V}{\varepsilon_{F}},
$$

where $n_{0} \approx v_{F} \varepsilon_{F}$ is the conduction electron density in the normal metal $\left(v_{F}\right.$ is the electron density of states at the Fermi level $\varepsilon_{F}$ ). Therefore the total number of "hot" electrons in the normal-metal side of the contact is

$$
\delta n=\gamma_{\mathrm{tr}}\left(n_{0} \Omega_{P C}^{(2)}\right) \frac{e V}{\varepsilon_{F}}, \quad \gamma_{\mathrm{tr}}=\frac{\beta_{\uparrow}^{(2)}+\beta_{\downarrow}^{(2)}}{4} .
$$

As can be seen from Eq. (26) the induced magnetic moment corresponding to the net spin density accumulated in the same region is

$$
\delta M=\mu_{B} \beta_{\mathrm{tr}}\left(n_{0} \Omega_{P C}^{(2)}\right) \frac{e V}{\varepsilon_{F}}, \quad \beta_{\mathrm{tr}}=\frac{\beta_{\uparrow}^{(2)}-\beta_{\downarrow}^{(2)}}{4} .
$$

Notice, that the ratio $\beta_{\operatorname{tr}} / \gamma_{\operatorname{tr}}$ determines the effective spin of an injected electron,

$$
S=\frac{\delta n_{\uparrow}-\delta n_{\downarrow}}{\delta n}=\frac{\beta_{\uparrow}^{(2)}-\beta_{\downarrow}^{(2)}}{\beta_{\uparrow}^{(2)}+\beta_{\downarrow}^{(2)}},
$$

and, therefore, is a measure of the spin polarization of the "hot" electrons.

Thus the spin accumulation inside PC by the applied voltage $V$ produces an additional magnetic moment inside the PC. If $d$ is of the size of several hundred $\mathrm{nm}$ and $\beta_{\mathrm{tr}} \approx 0.3$, which corresponds to a nearly ballistic $\mathrm{PC}, l \sim d$, and the spin polarization of $30 \%$ at the ferromagnetic/nonmagnetic $(\mathrm{F} / \mathrm{N})$ interface, one has

$$
\delta M \sim 10^{6} \mu_{B} \frac{e V}{\varepsilon_{F}} ; \quad \delta n \sim 10^{9} \frac{e V}{\varepsilon_{F}} .
$$

As one sees from Eq. (24), the dependence of $\beta_{\sigma}^{(2)}$ on the spin polarization is much weakened if $\kappa_{\sigma}^{(s)} \ll 1$, which prevents spin accumulation.

\section{Photocurrent}

As the photon momentum is orders of magnitude smaller than that of electrons in metals the change of the electron momentum under electron-photon scattering can be neglected and hence the main contribution to the photocurrent flowing through the point contact under electromagnetic irradiation is due to the changes in the electron density of states produced by vertical spin-flip transitions (in the energy-momentum space) by the photon field (see Fig. 1).

In order to find the photocurrent we first solve the Boltzmann equation (12) for the photon-induced change $f_{\sigma \mathbf{p}, 1}^{(s)}(\mathbf{r})$ in the electron distribution function. We do so to lowest (linear) order in the small parameter $d / l_{\mathrm{ph}}$ and with the boundary conditions $f_{\sigma \mathbf{p}, 1}^{(1,2)}(z=\mp L / 2)=0$. The matching conditions at the $\mathrm{F} / \mathrm{N}$ interface are given by Eq. (19) with the change $f_{\sigma \mathbf{p}}^{(s)} \rightarrow f_{\sigma \mathbf{p}, 1}^{(s)}$. Using these solutions one finds the photocurrent as

$$
\begin{gathered}
I_{\mathrm{ph}}=e \sum_{s=1,2} \int_{\Omega_{P C}^{(s)}} d \mathbf{r} \int \frac{d \mathbf{p}}{(2 \pi \hbar)^{3}} \times \\
\times\left[\alpha_{\uparrow,-\mathbf{p}}^{(s)}(\mathbf{r})-\alpha_{\downarrow,-\mathbf{p}}^{(s)}(\mathbf{r})\right] w_{\mathrm{ph}}^{(s)}\left\{f_{\uparrow \mathbf{p} 0}^{(s)}, f_{\downarrow \mathbf{p} 0}^{(s)}\right\} .
\end{gathered}
$$

Using Eqs. (23) and (24) in Eq. (31) one obtains the total current $I(V)$ in a diffusive point contact under irradiation as

$$
\begin{gathered}
I(V)=\frac{V}{R}+j_{\mathrm{ph}}(V) \\
j_{\mathrm{ph}}(V)=-\frac{\Delta R_{1}}{R_{1}^{2}}\left(V-V_{1}^{*}\right)+\frac{\Delta R_{2}}{R_{2}^{2}}\left(V-V_{2}^{*}\right) .
\end{gathered}
$$

Here $R_{s}(s=1,2)$ is the "dark" contact resistance due mainly to the impurities, while the relative change of the point-contact resistance caused by the irradiation is 


$$
\begin{aligned}
\frac{\Delta R_{s}}{R_{s}} & =\frac{\left(4 \pi \beta_{\mathrm{tr}}^{(s)}\right)^{2}}{3} \frac{c}{v_{F}} \frac{\left|W_{s}\right|^{2}}{\varepsilon_{F} \hbar \omega}\left(n_{0} \Omega_{P C}^{(2)}\right)\left(\frac{2 e^{2}}{h} R\right) \times \\
& \times \arctan \frac{2 \xi}{1-\xi^{2}+\left[\left(\hbar \omega-2 I_{s}\right) / \hbar v_{s f}\right]^{2}},
\end{aligned}
$$

where $\quad \beta_{\text {tr }}^{(s)}=\left|\beta_{\uparrow}^{(s)}-\beta_{\downarrow}^{(s)}\right| / 4, \quad e V_{s}^{*}=(3 / 4) \hbar \omega / \beta_{\text {tr }}^{(s)}$ and $\xi=\omega v_{F} / c v_{s f}$. According to Eq. (32) the dependence of the photocurrent on the irradiation frequency has a sharp peak corresponding to the resonant interaction of the electron spin and the electromagnetic field (see Fig. 3).

A comparison between Eq. (31) and the rate equation for photons generated by electronic spin-flip transitions induced by the electromagnetic field (see Ref. 10),

$$
\frac{d n_{\mathrm{ph}}^{(s)}}{d t}=-\int w_{\mathrm{ph}}^{(s)}\left\{f_{\uparrow \mathbf{p}}^{(s)}, f_{\downarrow \mathbf{p}}^{(s)}\right\} \frac{d^{3} \mathbf{p}}{(2 \pi \hbar)^{3}},
$$

where $n_{\mathrm{ph}}$ is the photon density, shows that the photocurrent may be rewritten in the form

$$
I_{\mathrm{ph}}=-e \sum_{s=1,2} \int_{\Omega_{s}} d \mathbf{r}\left(\left\langle\alpha_{\uparrow, \mathbf{p}}^{(s)}\right\rangle-\left\langle\alpha_{\downarrow, \mathbf{p}}^{(s)}\right\rangle\right) \frac{d n_{\mathrm{ph}}^{(s)}}{d t},
$$

which makes it clear that its magnitude depends on the net rate of photon absorption/emission in combination with the spin dependence of the effective transparency of the point contact. From Eq. (32) one notes that the microwaveinduced current changes sign at $V=V^{*}$, i.e., when the rate of photon emission by "hot" electrons begins to exceed the rate of photon absorption. (For the sake of definiteness, here and below we consider the case that the resonance takes place in metal 2 and we also drop the subscript $s=2$ ).

The close association between the electron transport and photon radiation processes allows us to express the photocurrent in terms of the power of emission and absorp-

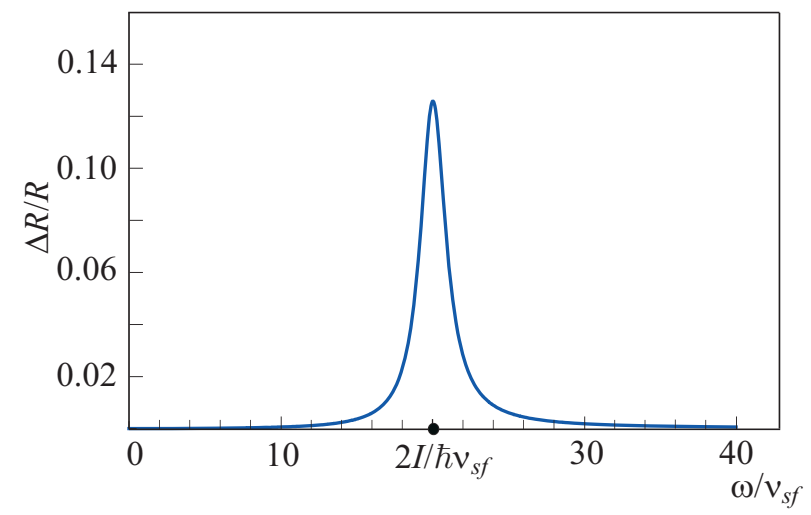

Fig. 3. Dependence of the relative resistance change under irradiation on the irradiation frequency $\omega$ normalized by the electron spin-flip relaxation frequency $v_{s f}$ for $v_{s f} / I=10^{-1}$ (here $I$ is the exchange energy). tion of photons by electrons in the point contact. Using Eqs. (17), (20), and (23) one finds that the net emitted power due to resonant $\left(\hbar \omega=2 I_{S}\right)$ absorption and emission of photons in the irradiated point contact, defined as $P(V)=\hbar \omega \int d \mathbf{r} d n_{\mathrm{ph}} / d t$, can be expressed as

$$
P(V)=P_{0}\left(-1+\frac{3}{2} \frac{V}{V^{*}}\right) .
$$

Here

$$
P_{0}=\frac{\pi}{2} \frac{c}{v_{F}} \frac{\omega}{\varepsilon_{F}}\left(n_{0} \Omega_{P C}^{(2)}\right)|W|^{2} \arctan \frac{2 \xi}{1-\xi^{2}}
$$

is the absorbed power due to photon absorption, while the second term in Eq. (36) is the emitted power due to photon emission from the point contact.

Comparing Eqs. (32) and (37) one finds that

$$
j_{\mathrm{ph}}(V)=\frac{3}{4} \frac{V-V^{*}}{V^{* 2}} P_{0},
$$

which makes it possible to find the power $P_{0}$ absorbed from the electromagnetic field by measuring $d j_{\mathrm{ph}}(V) / d V$ (see Eq. (32)) after first having determined $V^{*}$ from the condition $j_{\mathrm{ph}}\left(V^{*}\right)=0$. Furthermore, the net emitted power $P(V)$ can be determined by measuring $j_{\mathrm{ph}}(V)$ with the help of Eqs (38) and (36).

It follows from Eq. (36) that the radiation field is enhanced by the stimulated emission of photons if the proportionality coefficient is greater than zero, that is at $V>(2 / 3) V^{*}$. From here it follows that the point contact may be considered as an active element with the increment of the electromagnetic field given by

$$
\gamma=3 \pi^{2}\left(\frac{c}{v_{F}}\right)^{3} \frac{n_{0}}{\varepsilon_{F}} \mu_{B}^{2}\left(\frac{V}{V^{*}}-\frac{2}{3}\right) \arctan \frac{2 \xi}{1-\xi^{2}} .
$$

Inserting typical parameters for the metal electrodes, one finds $\gamma \sim 10\left(V / V^{*}-2 / 3\right)$ for $\hbar v_{s f} / I=10^{-1}$, and $V / V^{*}$ can reach as high as $\approx 10^{3}-10^{4}$ in realistic structures; therefore, the optical gain $g_{\text {opt }}=\gamma \omega / c$ can reach values $g_{\text {opt }} \sim 10^{6}-10^{7} \mathrm{~cm}^{-1}$. This exceeds the increment and the optical gain that can be achieved in conventional semiconductor lasers by orders of magnitude and hence an array of such magnetic point contacts can be used as the active area of powerful lasers [14].

\section{Conclusions}

In conclusion we have shown that by employing a ferromagnetic metal point contact as the optically active medium in a laser device the frequency of the generated coherent light can be in the desirable terahertz region while at the same time the optical gain can be increased by several orders of magnitude compared to conventional semiconductor laser materials. A possible problem is a too strong probability for a nonradiative decay of the population in- 
version of spin-split electron energy levels through the excitation of magnons. In order to avoid this problem we suggest to use ferromagnets with a sufficiently low magnetic exchange energy (Curie temperature) so that the electron-magnon relaxation length exceeds the point-contact diameter.

We emphasize that the theoretical approach used here is only valid in the weak electron-photon scattering limit and hence not strictly applicable if the intensity of the electromagnetic field is too high. Nevertheless, a preliminary analysis shows that the inverse spin population is preserved and that coherent laser light is generated also in this limit. However, since in this case many electron-photon scattering events can take place as an injected electron moves diffusively back and forth in the contact region, Eqs. (34)-(38) which relate the rate of photon generation to the electronic transport characteristics do not hold. A new theoretical approach should be developed in this limit of intensive electron-photon scattering which should assist the analysis of strong laser generation in ferromagnetic point contacts.

\section{Acknowledgments}

Financial support from the European Commission (FP7-ICT-FET-225955 STELE), the Swedish VR, and the Korean WCU program funded by MEST/NFR (R31-2008000-10057-0) is gratefully acknowledged.

1. I.O. Kulik, R.I. Shekhter, and A.N. Omelyanchouk, Solid State Commun. 23, 301 (1977).

2. A.N. Omelyanchouk, I.O. Kulik, and R.I. Shekhter, JETP Lett. 25, 437 (1977).

3. R.I. Shekhter and I.O. Kulik, Fiz. Niz. Temp. 9, 46 (1983) [Sov. J. Low Temp. Phys. 9, 22 (1983)].

4. Y.G. Naidyuk and I.K. Yanson, Point-Contact Spectroscopy Springer Series in Solid-State Sciences 145 Springer, New York (2005).

5. J.C. Slonczewski, J. Magn. Magn. Mater. 159, L1 (1996); ibid. 195, L261 (1999).

6. L. Berger, Phys. Rev. B 54, 9353 (1996).

7. A.M. Kadigrobov, R.I. Shekhter, M. Jonson, and V. Korenivski, Phys. Rev. B 74, 195307 (2006).

8. A.M. Kadigrobov, S. Andersson, D. Radić, R.I. Shekhter, M. Jonson, and V. Korenivski, J. Appl. Phys. 107, 123706 (2010).
9. A.M. Kadigrobov, S. Andersson, H.-C. Park, D. Radić, R.I. Shekhter, M. Jonson, and V. Korenivski, J. Appl. Phys. 111, 044315 (2012).

10. A. Kadigrobov, Z. Ivanov, T. Claeson, R.I. Shekhter, and M. Jonson, Europhys. Lett. 67, 948 (2004).

11. A.M. Kadigrobov, R.I. Shekhter, S.I. Kulinich, M. Jonson, O.P. Balkashin, V.V. Fisun, Yu.G. Naidyuk, I.K. Yanson, S. Andersson, and V. Korenivski, New J. Phys. 13, 023007 (2011).

12. Yu.G. Naidyuk, O.P. Balkashin, V.V. Fisun, I.K. Yanson, A.M. Kadigrobov, R.I. Shekhter, M. Jonson, V. Neu, M. Seifert, S. Andersson, and V. Korenivski, New J. Phys. 14, 093021 (2012).

13. A.M. Kadigrobov, R.I. Shekhter, I. Aronov, S.I. Kulinich, A. Pulkin, and M. Jonson, Fiz. Nizk. Phys. 37, 1163 (2011) [Low Temp. Phys. 37, 925 (2011)].

14. R.I. Shekhter, A.M. Kadigrobov, M. Jonson, E.I. Smotrova, A.I. Nosich, and V. Korenivski, Opt. Lett. 36, 2381 (2011).

15. Yu.V. Guliaev, P.E. Zilberman, A.I. Krikunov, A.I. Panas, and E.M. Epstein, JETP Lett. 85, 160 (2007).

16. X.R. Wang, Res. Lett. Phys., Article ID, 434936 (2008).

17. S.V. Vonsovskii, Magnetism, Wiley, New York (1974), Vol. 2, Ch. 3, p. 635; H.J. Zeiger and G.W. Pratt, Magnetic Interactions in Solids, Clarendon Press, Oxford (1973).

18. E.I. Rashba, Phys. Rev. B 62, R16267 (2000).

19. R.M. Potok, J.A. Folk, C.M. Markus, and V. Umanski, Phys. Rev. Lett. 89, 266602 (2002).

20. S.H. Chun, S.J. Potashnik, K.C. Ku, P. Schiffer, and N. Samarth, Phys. Rev. B 66, 100408 (2002).

21. A.T. Hanbicki, B.T. Jonker, G. Itskos, G. Kioseoglou, and A. Petrou, Appl. Phys. Lett. 80, 1240 (2002).

22. Yu.V. Gulyaev, P.E. Zilberman, I.V. Malikov, A.I. Panas, S.G. Chigarev, and E.M. Epstein, Pis'ma v ZhETF 93, 289 (2011).

23. Electromagnetic radiation from a planar contact between a ferromagnetic and a nonmagnetic semiconductor in an external magnetic field was detected, N.A. Viglin, V.V. Osipov, A.A. Samokhvalov, and O.G. Reznitskikh (eds.), Phys. Low-Dimens. Str. 1-2, 89 (1997).

24. I.O. Kulik, A.N. Omel'yanchuk, and R.I. Shekhter, Fiz. Nizk. Temp. 3, 1543 (1977) [Sov. J. Low Temp. Phys. 3, 740 (1977)].

25. I.O. Kulik, R.I. Shekhter, and A.G. Shkorbatov, Sov. Phys. JETP 54, 1130 (1981). 